\title{
Non solo le guerre. Sulla rilevanza storica delle "altre" catastrofi
}

\author{
di Matteo Pasetti
}

Feb 5, 2021 | Emergenza e (nuova) normalità, Emergenza/Normalità, In evidenza | $\underline{0}$ |

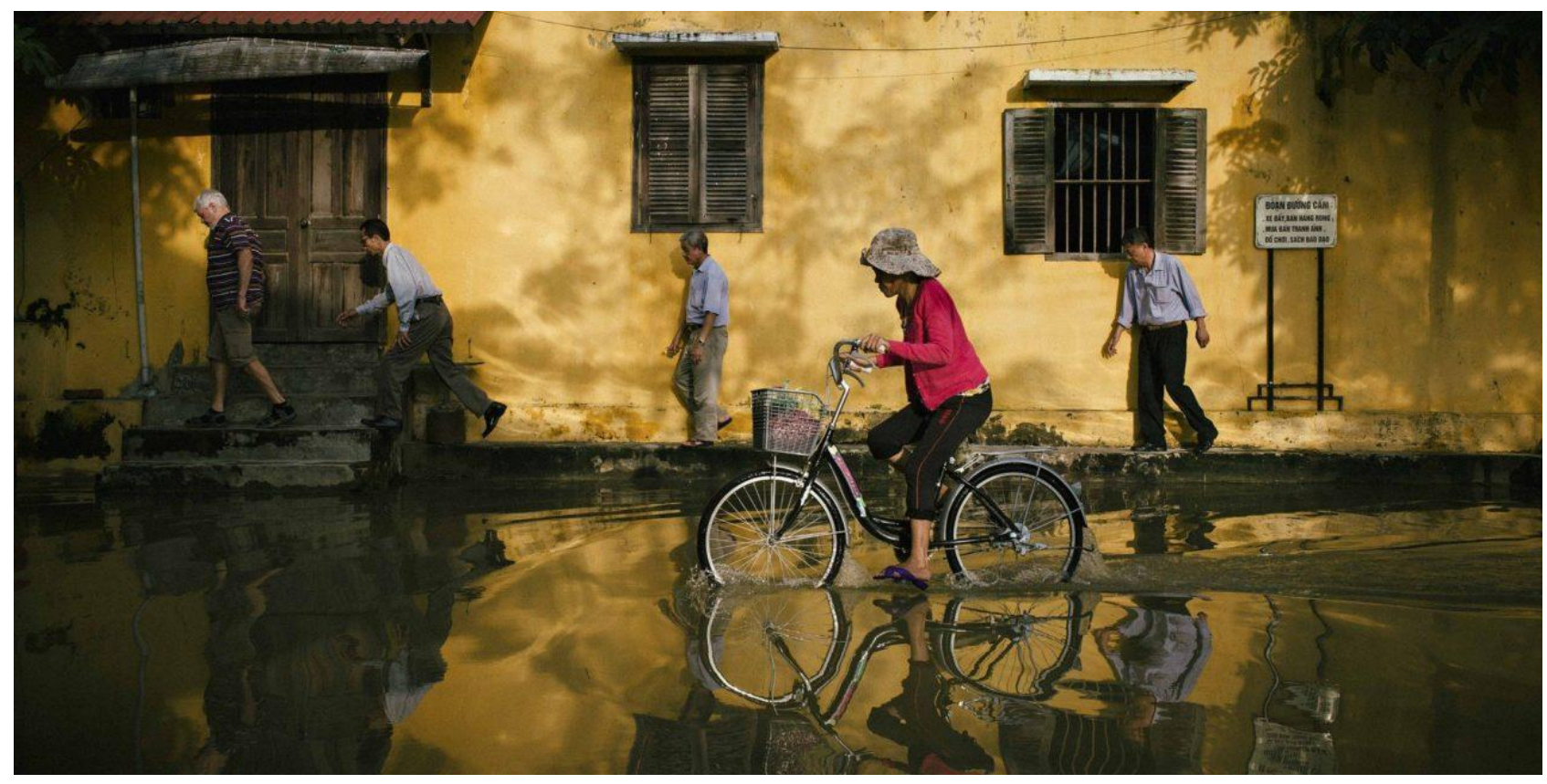

\section{Il dossier sulla Summer school 2020 si compone di 5 coppie di parole chiave. Questo testo afferisce alla coppia di parole chiave Emergenza/Normalità}

\begin{abstract}
Nella prima giornata della Summer School 2020 abbiamo chiesto a Matteo Pasetti e Patrizia Gabrielli di riflettere con noi sulle parole chiave Emergenza e Normalità. In questo articolo non ci occuperemo di guerre e nemmeno di altre categorie di avvenimenti tragici, come quelli scaturiti da insurrezioni e conflitti politici, da atti terroristici o criminali, oppure dal collasso di sistemi istituzionali o economici. Prenderemo in considerazione, invece, altre tipologie di situazioni emergenziali, che hanno origine nella complessità, nell'instabilità e nella fragilità del rapporto tra l'uomo e la natura.

In primo luogo proveremo a delineare un quadro d'insieme, distinguendo tra catastrofi "naturali", "tecnologiche" e "sanitarie". Successivamente sposteremo l'attenzione sulla loro rilevanza storica nel periodo che va dalla metà del XX secolo a oggi, soffermandoci in particolare su tre snodi cruciali: la dialettica tra stato e società civile; il ruolo del sistema mediatico; l'importanza della memoria.
\end{abstract}


Quando si fa riferimento alla storia, il binomio emergenza/ricostruzione è di solito associato in prima battuta ai grandi eventi bellici, ovvero agli sconvolgimenti da essi provocati, alle loro molteplici ripercussioni, ai problematici tentativi di ripristinare la normalità. Non è un caso, infatti, che di fronte a un qualsiasi disastro di dimensioni rilevanti il parallelismo scatti quasi in automatico: «È come una guerra!».

Se circoscriviamo la riflessione al Novecento, in particolare la Seconda guerra mondiale ha assunto da questo punto di vista un valore paradigmatico, venendo interpretata - per varie e fondate ragioni - come l'avvenimento in assoluto più traumatico nella storia globale del XX secolo. Di un secolo, cioè, che ha sancito il primato della politica sulla natura come principale artefice di traumi catastrofici per l'umanità[1].

Tuttavia, l'età contemporanea - e più in generale, l'intera storia dell'umanità - ha conosciuto altri innumerevoli eventi o fenomeni di estrema criticità, che, seppure su scale geografiche e temporali differenti, sono stati percepiti, affrontati e vissuti come vere e proprie emergenze.

Non solo alla guerra va riconosciuto, dunque, lo status di situazione emergenziale. Né soltanto i periodi postbellici hanno richiesto grandi opere di ricostruzione - materiale, ma anche politica, economica, morale - delle società umane; e tale constatazione appare ancor più evidente nel corso dell'attuale pandemia di Covid-19.

Per trattare del tema emergenza/ricostruzione in chiave storica non ci occuperemo, dunque, di guerre, e nemmeno di altre categorie di avvenimenti tragici, come quelli scaturiti da insurrezioni e conflitti politici, da atti terroristici o criminali, oppure dal collasso di sistemi istituzionali o economici. Tutti fenomeni che hanno una chiara ed esclusiva connotazione antropica. Prenderemo in considerazione altre tipologie di situazioni emergenziali, che hanno origine nella complessità, nell'instabilità e nella fragilità del rapporto tra l'uomo e la natura. In primo luogo, pur senza pretese tassonomiche, proveremo a delineare un quadro d'insieme, distinguendo tra catastrofi "naturali", "tecnologiche" e "sanitarie", ma sottolineando anche qualche intrinseco elemento in comune. Successivamente sposteremo l'attenzione sulla loro rilevanza storica nel periodo che va dalla metà del XX secolo a oggi, soffermandoci in particolare su tre snodi cruciali: la dialettica tra stato e società civile; il ruolo del sistema mediatico; l'importanza della memoria.

\section{Le catastrofi "naturali"}

La categoria delle catastrofi "naturali" comprende i disastri provocati da tutta una serie di calamità come terremoti, eruzioni vulcaniche, alluvioni, inondazioni, ecc... La differenza principale di tali catastrofi rispetto alle emergenze belliche, e la ragione per cui vengono definite "naturali", consiste nella loro genesi, cioè nel fatto che non hanno cause strettamente o solamente antropiche come le guerre. Con ogni evidenza non è l'uomo a scatenare un uragano, un'esplosione vulcanica, o una scossa tellurica. Ciononostante, come diversi studiosi hanno puntualizzato, è più corretto inserire l'aggettivo "naturali" tra virgolette[2].

Sebbene qualsiasi evento estremo sia potenzialmente devastante, esso dà origine a catastrofi soltanto nei casi in cui interagisce con la presenza umana sul pianeta, causando vittime e danni materiali ingenti. Come ha scritto lo scienziato inglese Karl Western: 
da un certo punto di vista, un disastro diventa un disastro solo quando vengono coinvolti uomini o ambienti creati dagli uomini. Una valanga in una valle disabitata o un terremoto in Artide sono eventi geofisici, non sono disastri.[3]

In altri termini, è l'impatto dei cataclismi sull' ecumène a produrre catastrofi "naturali". Ogni pericolo "naturale" è sempre collegato all' attività sociale dell'uomo[4]. E questa interazione tra agenti distruttivi e comunità umane, presenta almeno due implicazioni sul piano concettuale.

\section{Fenomeni antropici e sociali}

In primo luogo è il grado di devastazione degli insediamenti umani colpiti, più che la potenza del fenomeno in quanto tale, a determinare la gravità dell'evento (prendendo il caso dei terremoti, si potrebbe affermare che ha maggior rilevanza la scala Mercalli di quella Richter)[5]. Da questa prospettiva palesemente antropocentrica, l'importanza di una calamità è stabilita quindi dalle conseguenze materiali e demografiche sull'ambiente umano, mentre non esiste un rapporto di proporzionalità diretta tra la forza intrinseca dell'agente distruttivo e la drammaticità dell'episodio.

Per esempio, tra i grandi terremoti dei nostri tempi, uno dei più catastrofici è stato quello del $2010 \mathrm{a}$ Port-au-Prince (Haiti) che, sebbene di magnitudo non eccezionale (7.0), ha causato almeno 230.000 morti (secondo altre stime, le vittime sarebbero state più di 300.000) e ha coinvolto circa 3 milioni di persone. Viceversa, il sisma più potente mai registrato dalle moderne strumentazioni - cioè quello di Valdivia (Cile) del 1960 che ha raggiunto una magnitudo di 9.5 - rappresenta un caso meno disastroso (restarono sotto le macerie non più di 3.000 persone), poiché localizzato in un'area a bassa densità di popolazione e con edifici costruiti prevalentemente in legno. Analogamente, pochi anni dopo, il terremoto in Alaska del 1964 (di magnitudo 9.2) ha provocato solo qualche decina di vittime, nonostante l'enorme energia sprigionata. Ciò non toglie che queste potenti scosse sismiche abbiano avuto effetti geofisici su scala globale, arrivando addirittura a imprimere lievi modifiche alla forma del pianeta[6]. Ma da un punto di vista antropocentrico il loro impatto è risultato meno devastante rispetto ad altri fenomeni catastrofici. Anche se si potrebbe obbiettare sulla liceità di stilare classifiche in base al numero di morti o all'entità dei danni materiali, il dato statistico resta ed ha un suo interesse.

D'altra parte, anche violentissimi movimenti tellurici lontani da centri abitati - per esempio con epicentro al largo di un oceano - e che hanno generato un gigantesco maremoto possono provocare catastrofi colossali. È ciò che è avvenuto il 26 dicembre 2004 con gli tsunami che si sono abbattuti sulle coste dell' intero sud-est asiatico e hanno causato circa 250.000 morti, mezzo milione di feriti e oltre tre milioni di sfollati.

In secondo luogo disastri e catastrofi "naturali" vanno interpretati anche come fenomeni sociali. Non solo perché implicano profondi sconvolgimenti nella struttura organizzativa, nelle dinamiche quotidiane, nel tessuto affettivo ed emotivo delle società colpite, ma anche perché si configurano in base alle specifiche vulnerabilità dei sistemi sociali. È l'interazione tra il tipo di agente distruttivo, il tipo di ambiente geo-storico e il tipo di società a modellare le forme della catastrofe. Un terremoto o un'alluvione possono cioè causare emergenze di grado differente secondo variabili che non riguardano soltanto la natura geomorfologica del luogo interessato, ma anche la densità abitativa, le condizioni urbanistiche, lo sviluppo tecnologico, le conoscenze scientifiche, e più in generale il sistema sociale costituitosi nel corso del tempo[7]. In tal senso, da un lato l'impatto più o meno disastroso di un evento estremo è determinato almeno in parte dal passato, dall'evoluzione della 
comunità colpita; e dall'altro produce effetti sociali di medio e lungo periodo. Il nesso con la storia è insomma complesso e biunivoco.

\section{Le catastrofi "tecnologiche"}

Le osservazioni precedenti inducono a sfumare la convenzionale distinzione tra catastrofi "naturali" e catastrofi "tecnologiche" (o "man-made", secondo la terminologia anglosassone). Con tale categoria s'intendono quei disastri derivati da attività umane, o più precisamente dipendenti dall'uso delle varie tecnologie sviluppate dall'uomo, come gravi incidenti chimici, nucleari o ingegneristici[8]. Nella storia del Novecento un caso paradigmatico è rappresentato dall'incidente di Černobil' del 1986, con il guasto avvenuto alla centrale nucleare sovietica e il conseguente disastro ambientale che se ne è generato e che è costato la vita, o ha causato tragici problemi di salute, a un numero imprecisato di persone e altri esseri viventi.

A ben vedere gli esempi di questo tipo sono assai più numerosi e comprendono tutte le emergenze originate da errori, imperizie, imprudenze dell'uomo: tra le più frequenti i crolli di dighe e le conseguenti inondazioni[9]. Nella loro molteplicità si tratta di disastri che non nascono dall'impatto di agenti esterni sulle comunità umane, bensì in conseguenza dei rischi assunti, in modo più o meno consapevole, nell'applicazione di conoscenze tecnico-scientifiche. Tuttavia, anche in questi casi, la catastrofe deriva dall'interazione tra diversi fattori: il tipo di tecnologia e il livello di pericolosità, certo, ma pure il contesto ambientale, le decisioni politiche, l'organizzazione sociale, le risorse economiche, i retaggi culturali, e così via. La complessità delle connessioni tra vari elementi di vulnerabilità è insomma all'origine delle catastrofi "tecnologiche", così come di quelle "naturali". La linea di demarcazione tra le une e le altre risulta molto labile.

Senza contare che a volte le due tipologie di disastri appaiono in stretta correlazione. Si prenda il caso della città giapponese di Fukushima, sede di una centrale nucleare che nel marzo 2011 è stata colpita da un terremoto di eccezionale magnitudo (9.0), al quale ha fatto seguito un devastante tsunami con onde superiori ai dieci metri d'altezza. La combinazione tra i due fenomeni "naturali" ha prodotto un impatto ben più forte dei livelli di sicurezza previsti nella progettazione della centrale facendo saltare gli impianti di raffreddamento di tre reattori e provocando la dispersione di radioattività. Essendo il Giappone un paese all'avanguardia sia nel campo delle costruzioni antisismiche, sia nella dotazione di strumenti di prevenzione, allarme e pronto intervento, 1' "incidente" di Fukushima non ha avuto gli esiti catastrofici che si sarebbero registrati altrove; d'altro canto il disastro nucleare è stato pur sempre grave, e soprattutto è la conseguenza non solo della combinazione tra eventi "naturali" e pericolo "tecnologico", ma anche della scelta politica di costruire una centrale in una zona ad altissimo rischio sismico. Fukushima rappresenta un perfetto esempio, insomma, della complessa interazione di fattori che sono all'origine di un'emergenza funesta.

\section{L'accelerazione dell'Antropocene}

La dicotomia tra catastrofi "naturali" e "man-made" appare ancor più inadeguata se introduciamo nella riflessione la nozione di Antropocene. Com'è noto, il termine è stato adottato nel 2000 dal chimico olandese Paul Crutzen (già Premio Nobel nel 1995) per definire un nuovo periodo nella storia della Terra, connotato dalla pressione delle attività umane sull' ecologia del pianeta e apertosi nel tardo Settecento con l'inaugurazione di un regime energetico basato sui combustibili fossili[10]. 
Dal 2000 in poi alle teorie di Crutzen hanno fatto seguito varie altre formulazioni e il concetto ha conosciuto crescente fortuna, non solo nella letteratura scientifica: negli ultimi anni, un certo interesse ha riscosso, ad esempio, l'Anthropocene Project, che attraverso mostre fotografiche in diversi paesi (tra cui, in Italia, al Mast di Bologna), pubblicazioni di stampo divulgativo e un documentario realizzato in collaborazione con il fotografo Edward Burtynsky, ha illustrato al grande pubblico alcuni effetti invasivi dell'irresponsabile impatto delle attività umane sull'ambiente[11]. Ma mentre le interpretazioni sulle origini dell'Antropocene divergono qualcuno le fa risalire addirittura alla scoperta del fuoco -, meno controversa è la tesi secondo cui l'influenza dell'uomo sul "sistema Terra" è cresciuta vertiginosamente dopo il 1945, raggiungendo livelli ormai insostenibili dal punto di vista ambientale. L'impennata demografica, l'inurbazione globale, l'aumento esponenziale dei consumi energetici, l'accumulo di anidride carbonica nell'atmosfera, l'invasione mondiale di merci di plastica sono soltanto alcune delle dinamiche che dalla metà del XX secolo hanno impresso una «grande accelerazione» all' impatto dell'uomo sul pianeta[12].

Questo insieme di processi ha avuto tra le sue conseguenze l'incremento di un certo tipo di disastri lenti e silenziosi - e non deflagranti come un terremoto - determinati dalla sempre più complessa interazione tra le attività antropiche e i diversi ecosistemi. Il fenomeno più significativo è dato senza dubbio dall'emergenza climatica, che alcuni intellettuali considerano ormai irreversibile[13]. Il riscaldamento dell'atmosfera - causato soprattutto da una concentrazione di anidride carbonica cresciuta più di otto volte dal 1945 - ha aumentato le probabilità sia di siccità devastanti, sia di precipitazioni catastrofiche. Esempi eclatanti sono quello del Sahel (siccità e carestia) negli anni Settanta-Ottanta o del Golfo del Bengala (cicloni e inondazioni) nel 1970 e nel 1991. Ma per quanto sia difficile dimostrare la stretta consequenzialità dei singoli fattori - ovvero, un diretto rapporto tra certe attività umane reiterate nel tempo, l'innalzamento della temperatura globale e la desertificazione o l'inondazione di una precisa zona del mondo - nell'Antropocene le crisi ambientali sono sempre più gravi, diffuse e frequenti. E la loro crescente intensità rivela «la peculiarità dell'età contemporanea anche in termini ambientali»[14].

\section{Le catastrofi "sanitarie"}

L'attuale pandemia di Covid-19 ha riportato in primo piano l'emergenza "sanitaria", assai frequente nella storia dell'umanità, anche in età contemporanea. Senza bisogno di richiamare le ondate di peste dei secoli più remoti, è sufficiente ricordare le varie epidemie virali che hanno colpito il Novecento: dalla gravissima influenza nota come "Spagnola", che uccise decine di milioni di persone nel mondo tra il 1918 e il 1920, ai casi più recenti di Aviaria, Aids, Ebola, Sars, fino al "Coronavirus" dei giorni nostri[15]. Sono tutte malattie infettive originate per zoonosi, ovvero a causa di un salto di specie dell'elemento patogeno che a un certo punto della sua evoluzione riesce a trasferirsi da una data specie animale all'uomo[16], alle quali si può aggiungere tutta una serie di gravi infezioni batteriche, come la diffusione di tifo o colera (l'ultima importante epidemia di colera in Italia risale al 1973).

Le differenze fra queste malattie e fenomeni come terremoti o alluvioni sono piuttosto evidenti e riguardano innanzitutto la dimensione spazio-temporale: avendo un carattere epidemico, se non pandemico, le emergenze sanitarie colpiscono ampie aree del mondo per un periodo di tempo più lungo. Sono inoltre parzialmente difformi i danni provocati, poiché le epidemie non causano distruzioni materiali (perlomeno non in modo diretto). E sono differenti i problemi che le società colpite devono affrontare, sia nella fase acuta della crisi sanitaria sia in quella della ripartenza e del ritorno alla normalità. Tuttavia, anche in questo caso, l'emergenza rimarca come l'esistenza umana 
sia intrecciata all'ambiente biologico, come l'uomo sia incorporato (embedded) nella natura, come la storia antropica non sia scindibile da quella del pianeta in cui si svolge.

L'avvento dell'Antropocene non ha spezzato il legame: se da un lato ha significato per l'uomo una maggior capacità di separare le proprie attività produttive dai ritmi della natura, dall'altro non ha consentito un effettivo affrancamento dai pericoli biologici. Anzi, l'alterazione degli equilibri ecosistemici ha accresciuto il rischio di crisi sanitarie, come dimostrano anche le pandemie degli ultimi decenni, in qualche misura correlate alle devastazioni ambientali e ai modelli sociali dominanti: «la distruzione degli ecosistemi - scrive David Quammen - sembra avere tra le sue conseguenze la sempre più frequente comparsa di patogeni in ambiti più vasti di quelli originari»[17], aumentando dunque la probabilità che qualche virus entri in contatto con l'uomo e sia in grado di adattarsi alla nuova specie.

\section{La miopia storiografica}

Differenti tipologie di emergenze "non belliche", dunque, si sono succedute nella storia, con un incremento sia di frequenza che di gravità nell'epoca segnata dalla «grande accelerazione» dell'Antropocene. Ciononostante gli storici dell'età contemporanea hanno mostrato solo una sporadica o altalenante attenzione per tali fenomeni, spesso trascurati se non esclusi del tutto dai propri orizzonti di indagine. Di fatto, le crisi ecologiche e gli altri grandi eventi catastrofici sono entrati di rado nelle narrazioni storiche dedicate al Novecento (per non parlare della manualistica e della didattica scolastica) e tutt'al più sono stati confinati in un campo di ricerca autonomo - la storia ambientale - relegata in posizione defilata nelle complessive ricostruzioni dei processi sociali, economici, politici. In un suo recente volume Gabriella Gribaudi ha individuato la probabile ragione di fondo di tale lacuna nel fatto che «i disastri "naturali" vengono considerati incidenti e per questo al di fuori del controllo o dell'azione umana e quindi il loro studio viene lasciato interamente alle scienze dure»[18].

È avvenuto insomma qualcosa di simile a ciò che lo scrittore indiano Amitav Ghosh ha riscontrato in merito alla scarsa presenza del cambiamento climatico e dei suoi effetti tra i temi della letteratura contemporanea; come se quest'ultima fosse stata colpita da una forma di «grande cecità» - o di «grande alienazione», per tradurre alla lettera il titolo originale del libro, The Great Derangement verso uno dei principali problemi dell'epoca odierna[19]. Ecco, se la storiografia non è stata del tutto cieca, perlomeno è stata miope e ha faticato a mettere a fuoco certi fenomeni nel quadro complessivo dell'età contemporanea.

\section{Eventi di rottura}

Resta il fatto che, alla pari dei conflitti bellici, anche le altre catastrofi possono avere significative ripercussioni sul piano storico, non solo per l'impatto sulla demografia e/o sull'habitat umano, ma pure sul tessuto sociale, sull'economia, e talvolta anche sulla politica. Si tratta cioè di fenomeni che innalzano il tasso di mortalità ben oltre i livelli ordinari e che in alcuni casi - terremoti e alluvioni, in particolare - distruggono infrastrutture, edifici, abitazioni, beni e oggetti della vita quotidiana. Un genere di devastazione che presenta forti analogie con i danni provocati dalle guerre: si pensi, come esempio più lampante, ai bombardamenti aerei della Seconda guerra mondiale[20]. Le conseguenze sociali ed economiche sono ovviamente profonde, e lo sono spesso anche nel caso delle emergenze che non producono distruzioni materiali, come nel caso di crisi ambientali e pandemie. Si tratta 
dunque di fenomeni che, sebbene in modalità differenti rispetto ai conflitti bellici, possono indurre mutamenti politici: non è raro che a una catastrofe faccia seguito una crisi di regime, come è avvenuto in Nicaragua dopo il terremoto del dicembre 1972 che destabilizza la dittatura dei Somoza; o perfino una crisi dell'ordine geopolitico, come nel subcontinente indiano, con l'insurrezione, la guerra civile e la successiva proclamazione dello stato indipendente del Bangladesh provocate dall'epocale alluvione del 1970 nel Golfo del Bengala.

A volte la cesura che l'evento traumatico imprime nella vita di una comunità viene amplificata dalle modalità stesse con cui il potere politico conduce la ricostruzione. Diversi casi di studio offrono uno spaccato di grande interesse da questo punto di vista, evidenziando il carattere autoritario e centralistico di certi sistemi statali. Come è avvenuto a Tashkent in Uzbekistan, sotto il regime sovietico: qui, dopo il terremoto del 1966, la città è stata ricostruita secondo un modello culturale e urbanistico del tutto privo di legami col passato, sacrificando ciò che restava dei quartieri tradizionali, ridisegnando completamente la topografia, imponendo un nuovo stile architettonico e urbanistico con l'obiettivo di creare «la città modello della modernità sovietica nelle periferie arretrate»[21]. Anche altrove, e in scenari storici diversissimi tra loro, si sono attuati progetti di ricostruzione che hanno trasformato profondamente il territorio rispetto al passato. È il caso di un paese come Gibellina, in Sicilia, letteralmente traslocato a chilometri di distanza dall'insediamento originario dopo il sisma del 1968 nella valle del Belice. Oppure della ricostruzione avviata nello stato indiano del Gujarat, in seguito al devastante terremoto del gennaio 2001, condotta tramite forme di finanziamento miste - private e pubbliche, governative e internazionali - che hanno perseguito un progetto di riedificazione urbana molto distante dalle tradizioni abitative dell'antico sistema castale, suscitando non poche resistenze tra le popolazioni locali[22].

Le catastrofi, dunque, imprimono una rottura periodizzante su scale differenti. Innanzitutto segnano una cesura nella vita ordinaria delle persone e delle comunità investite dall'emergenza, per le quali il flusso del tempo si divide in "un prima" e "un dopo". La speranza di un ripristino della precedente "normalità" è destinata a rimanere disattesa e gli eventi acquisiscono spesso la valenza di vere e proprie svolte storiche, innescando mutamenti significativi in vari ambiti: dalla politica ambientale alla topografia urbanistica, dalla percezione culturale del rischio allo sviluppo tecnologico ed economico.

Il tema si presta perciò a essere indagato sotto molteplici angolazioni. Proveremo ora - mantenendo sullo sfondo la storia dell'Italia repubblicana costellata di una lunga sequenza di calamità - a introdurre alcuni spunti di riflessione su tre agenti di primo piano nello svolgimento dell'emergenza e della ripartenza: lo stato, i media, la memoria.

\section{Il ruolo dello stato}

Sia nel pieno della fase emergenziale sia nel passaggio a quella della ricostruzione o ripartenza, tutti i tipi di catastrofe inducono un processo di ridefinizione del rapporto tra pubblico e privato che si traduce in primo luogo nell'attribuzione di un ruolo cruciale allo stato. Di fronte all'emergenza sono le autorità e le istituzioni pubbliche ad avere il compito di assicurare i soccorsi e i primi interventi, poi di gestire l'uscita dalla crisi e il ritorno all'ordinario, e infine di predisporre soluzioni per evitare, per quanto possibile, il ripetersi delle tragedie, o perlomeno per ridurne la gravità; per esempio, attraverso la riforma dei quadri normativi in materia di prevenzione, il finanziamento della ricerca scientifica, l'investimento in strutture sanitarie o in altri settori specifici, la costruzione di appositi apparati per la gestione delle calamità, e così via. In breve nell'età contemporanea, connotata da una generale estensione delle funzioni statali, l'intervento dello stato in situazioni di 
forte criticità diventa essenziale; ma il suo ruolo appare altrettanto decisivo in absentia, cioè quando il sistema pubblico non riesce a fornire risposte e servizi adeguati, pregiudicando il superamento della crisi.

Se facciamo riferimento al caso italiano i terremoti del 1976 in Friuli e del 1980 in Irpinia hanno fornito due esempi antitetici di buona e cattiva gestione dell'emergenza, benché, paradossalmente, il modello operativo che è stato inizialmente messo in pratica era più o meno lo stesso. Mentre in Friuli la collaborazione tra i vari corpi e livelli istituzionali (governo centrale, commissario straordinario, esercito, ente regionale, amministrazioni locali, ecc.) ha funzionato, permettendo «non solo di ridurre i tempi della ricostruzione [...], ma di rilanciare l'economia, forte delle rinnovate infrastrutture realizzate dallo stato», l'Irpinia è diventata «il simbolo di una pessima gestione delle emergenze civili»[23]. Già nella fase drammatica del dopo-sisma si era registrato un grave ritardo dei soccorsi e degli aiuti, poi, nel periodo successivo, si sviluppa un intreccio perverso tra politica, affarismo e malavita. Le cospicue risorse investite per rilanciare l'economia meridionale finiscono nelle mani di consorterie locali, di organizzazioni camorristiche e di imprenditori senza scrupoli (anche del Nord)[24]. D'altra parte, come ha osservato lo storico Piero Bevilacqua, fin dai terremoti di inizio Novecento i lavori e i finanziamenti pubblici sono diventati in Italia lo strumento per eccellenza dell'intervento statale straordinario, funzionando come «occasione di ridistribuzione dall'alto della ricchezza», ma con la finalità di ripristinare il sistema di potere e l'ordine sociale preesistenti, più che di agire in profondità per rimodellare $\mathrm{i}$ rapporti di classe, ridurre gli squilibri, sanare il tessuto economico e civile[25].

La reazione agli eventi catastrofici rappresenta quindi una sorta di "cartina tornasole" per valutare modalità e capacità di funzionamento di una macchina statale, ma anche per riflettere sull'evoluzione dei rapporti tra potere politico e società civile. Tanto più che, come spiegano vari studi di psicologia sociale, l'atteggiamento delle comunità colpite da un trauma muta nel tempo, secondo una logica centrifuga: mentre nel momento immediatamente successivo all'esplosione dell'emergenza prevale la tendenza alla solidarietà, alla cooperazione, alla collaborazione con gli attori istituzionali, in seguito emergono amarezza, disillusione, individualismo, opportunismo, rancore, talvolta anche rabbia, con un' escalation che può sfociare in comportamenti sociali violenti[26]. È facile assistere quindi al rapido riaffiorare delle divisioni sia tra gli schieramenti politici, sia all'interno del corpo sociale, con una progressiva polarizzazione tra interessi privati e pubblici. In tal modo lo stato, al quale inizialmente si affidano le speranze di una pronta ripartenza, può trasformarsi in capro espiatorio.

\section{Il sistema mediatico}

Sulla dialettica tra pubblico e privato esercita una pesante pressione anche il sistema mediatico. L'insieme dei mezzi di comunicazione di massa offre innanzitutto una narrazione degli eventi che influisce fortemente sull'orientamento dell'opinione pubblica. A seconda del grado di libertà di espressione concessa dal regime politico, questa narrazione può essere più o meno univoca $\mathrm{o}$ pluralista. Nell'Italia repubblicana, sebbene in un quadro di generale democraticità, la cronaca e l'interpretazione dei disastri sono state spesso subordinate all'appartenenza politica e svolte con intenti strumentali. Un esempio emblematico è quello del Vajont nel 1963, quando la quasi totalità delle testate giornalistiche accreditano la versione ufficiale, descrivendo la sciagura come un evento naturale, imponderabile e ineludibile, causato esclusivamente dalla sfortuna: un pezzo di montagna che frana, cade nell'invaso di una diga e provoca un'inondazione sulla valle sottostante, travolgendo i centri abitati. Di fatto solo Tina Merlin, sulle pagine dell'organo di stampa del Partito comunista «l'Unità», decide di battersi per svelare la verità e denunciare la responsabilità di chi 
aveva voluto a tutti i costi realizzare un'opera ingegneristica ad alto rischio, ignorando le opposte opinioni[27]. Poi, ma solo a distanza di trent'anni, è stato uno spettacolo teatrale trasmesso in televisione a divulgare a un ampio pubblico la realtà di un disastro tutt'altro che naturale[28].

In tempi più recenti, con l'avvento di Internet e le trasformazioni che la diffusione della rete ha imposto anche nel settore dell'informazione mediatica, pure la narrazione delle catastrofi ha conosciuto profonde mutazioni, frantumandosi in una miriade di flussi comunicativi. In Italia come nel resto del mondo, gli stessi media tradizionali sono costretti in qualche misura a un ruolo subalterno. Ciò ha generato fra l'altro un problema di ipertrofia dell'informazione che talvolta ostacola la circolazione di notizie fondate e attendibili. In altri termini mentre è sempre più alla portata di molte persone approfondire la conoscenza su eventi emergenziali che avvengono in qualsiasi luogo del pianeta, è diventato più difficile sia orientarsi nel caos di informazioni che circolano in rete sia distinguere quelle seriamente documentate dai falsi. Si tratta di un aspetto che ha conseguenze di rilievo sulla formazione delle opinioni dei singoli individui, nonché sui comportamenti degli stessi cittadini che si trovano a vivere situazioni di crisi.

La questione delle responsabilità della comunicazione mediatica nell'evoluzione stessa delle emergenze è emersa chiaramente anche nel corso della catastrofe "sanitaria" provocata dalla pandemia di Covid-19, ma è ormai da tempo che si è acceso il dibattito sul ruolo dei media e in particolare sulla tendenza alla spettacolarizzazione delle catastrofi. In un celebre saggio pubblicato una ventina di anni fa, Susan Sontag si interrogava proprio su questa pratica di mettere in scena il «dolore degli altri» e sulle conseguenti reazioni emotive del pubblico:

Assistere da spettatori a calamità che avvengono in un altro paese è una caratteristica ed essenziale esperienza moderna, risultato complessivo delle opportunità che da oltre un secolo e mezzo ci offrono quei turisti di professione altamente specializzati noti come giornalisti. [...] "Il sangue in prima pagina" recita la collaudata linea guida dei tabloid e dei notiziari televisivi che danno informazioni flash ventiquattr'ore su ventiquattro - di fronte ai quali reagiamo con compassione, indignazione, curiosità o approvazione, man mano che ciascuna miseria ci si para dinanzi agli occhi.[29]

Anche se Sontag si riferiva in particolare «alla costante crescita dei flussi di informazione sui tormenti della guerra», le sue parole descrivono nondimeno l'atteggiamento mediatico nei confronti delle catastrofi "non belliche". Per citare il titolo di un altro fondamentale saggio sul tema, è «lo spettacolo del dolore» ad attirare l'attenzione del pubblico, e quindi a offrire una risorsa di primaria importanza per la comunicazione mediatica[30].

Tuttavia, proprio perché prevalgono le leggi inesorabili dello spettacolo, l'attenzione mediatica sulle catastrofi ha una durata limitata e svanisce man mano che cala l'intensità della fase emergenziale. Le vittime vengono lentamente abbandonate e dimenticate e ciò che resta, semmai, è la reiterazione di stereotipi consolidati. Riprendendo l'esempio dei terremoti in Friuli e Irpinia, si scorge nel discorso mediatico prevalente la ripetizione di rappresentazioni dicotomiche che contrappongono la presunta efficienza nordista al cliché del meridionale inerte, ozioso, opportunista, corrotto, ignorante. Secondo un'interpretazione stereotipata che trova ancora circolazione nei discorsi mediatici, i fallimenti della ricostruzione irpina sarebbero insomma da imputare principalmente all'antropologia delle stesse vittime, incapaci di reagire e colpevoli dello sperpero di denaro pubblico[31]. Procedimenti di simile banalizzazione avvengono fra l'altro, su scala globale, anche nei confronti delle vittime di disastri in paesi più poveri, "arretrati", che vengono in un certo senso considerate corresponsabili di tragici eventi proprio a causa delle loro condizioni di vita materiale e culturale, secondo l'idea che "da noi", cioè nel mondo occidentale, "non sarebbe potuto accadere". 


\section{La memoria}

Un altro agente che gioca una funzione importante nella storia delle emergenze è la memoria. «Per certi aspetti - sostiene Gabriella Gribaudi - la memoria della catastrofe è simile a quella delle vittime della guerra aerea», poiché in un attimo si perde tutto[32]. Ogni esperienza traumatica di tale intensità inevitabilmente rimane impressa nei ricordi di chi l'ha vissuta. Anche le memorie private partecipano alla rappresentazione del disastro come punto di rottura, cesura nello svolgimento lineare della propria esistenza, operando un processo di mitizzazione del passato per cui c'era un prima in cui prevaleva l'armonia e poi il caos, la disperazione, il lutto.

Come nelle guerre, inoltre, accanto alla memoria individuale delle vittime e dei testimoni, e poi alla composita e spesso divisiva memoria pubblica, si stratificano le memorie volatili dei semplici spettatori che hanno seguito gli eventi attraverso i media. Si tratta di una varietà di memorie, spesso altrettanto ipertrofica delle narrazioni mediatiche, che difficilmente compone un discorso in grado di permeare l'opinione pubblica in modo univoco. Ma mentre le memorie "calde" post-belliche tendono a mantenere alto il tasso di conflittualità interno alle comunità - generando quel problema di «eccesso di memoria», di cui si è tanto argomentato[33] -, le memorie delle altre catastrofi tendono a "raffreddarsi", a rimanere vive e sensibili solo per le vittime. Si innesta, dunque, un progressivo e generale oblio - un problema di «memoria corta», ha concluso Salvatore Botta ricostruendo la casistica italiana[34] - che ha ripercussioni anche sulla prevenzione di nuovi episodi. Così, vari studi di antropologia dei disastri hanno messo in luce l'irrazionale ostinazione delle comunità umane a non abbandonare luoghi pericolosi, oppure a non provvedere alle dovute precauzioni, ignorando gli avvisi degli esperti e il ricordo di precedenti catastrofi. Come ha sottolineato Gianluca Ligi, «sono proprio questi orientamenti "culturali”, in senso antropologico, che plasmano la vulnerabilità sociale di una comunità rispetto a un certo tipo di evento estremo»[35].

Ed è proprio quando la memoria collettiva svanisce e perde la sua capacità di preservare la società dalle esperienze emergenziali già affrontate in passato che si fa più impellente il contributo richiesto alla ricostruzione e alla conoscenza storica.

Note:

[1] Cfr. M. Livi Bacci, I traumi d'Europa. Natura e politica al tempo delle guerre mondiali, Il Mulino, Bologna 2020.

[2] Sia nell'ambito delle scienze umane che in altri settori disciplinari, la letteratura sul concetto di disastro o catastrofe - due termini, fra l'altro, talvolta utilizzati con differenti sfumature semantiche - è ampia e stratificata nel tempo. Per qualche rassegna introduttiva al dibattito, si rinvia a A. G. Noto, La "disastrologia": approcci e contributi significativi, in «Storia e futuro», 17, 2008; G. Ligi, Antropologia dei disastri, Laterza, Roma-Bari 2009; F. Walter, Catastrofi. Una storia culturale, Angelo Colla, Vicenza 2009 (ed. or. 2008); W. G. von Hardenberg, A. F. Penna, A. F. Saba, Catastrofi, in «Passato e presente», 82, 2011. 
[3] K. A. Western, The Epidemiology of Natural and Man-made Disasters. The Present State of the Art, University of London, London 1972, citato in Ligi, 2009, p. 3.

[4] Categorico, in tal senso, il geologo Mario Tozzi, volto noto anche al pubblico televisivo per i suoi programmi divulgativi: «le calamità naturali non esistono, esistono solo il naturale divenire di un pianeta attivo e dinamico e la nostra incapacità di tenerne conto». M. Tozzi, Dal terremoto di Lisbona allo tsunami del Sudest asiatico: 250 anni di lotta tra l'uomo e la natura, Rizzoli, Milano 2005, p. 10.

[5] Com'è noto, mentre la scala Richter misura l'energia sprigionata da un terremoto nel punto di rottura della crosta terrestre (l'ipocentro), la scala Mercalli misura l'intensità di un sisma sulla base dei danni prodotti a persone e manufatti.

[6] Cfr. A. Piersanti, Gli effetti dei grandi terremoti sul pianeta terra, in «Silvæ», 7, 2007. Si tratta di un testo divulgativo firmato dall'allora direttore dell'Istituto nazionale di geofisica e vulcanologia (Ingv) di Roma 1.

[7] Per questa lettura socio-antropologica della nozione di disastro, cfr. Ligi, 2009, pp. 19-43, che si basa in particolare su alcuni studi classici come quelli di M. Barkun, E. Quarantelli, D. Wenger, K. Hewitt, A. Oliver-Smith.

[8] Sul rapporto tra i concetti di "naturale" e "man-made", cfr. E. Bianchi, Uomo, rischio, natura: considerazioni intorno allo stato presente della ricerca, in G. Botta (a cura di), Eventi naturali oggi. La geografia e le altre discipline, Cisalpino, Milano 1993.

[9] Cfr. A. F. Saba, Le dighe, in «Passato e presente», 82, 2011.

[10] L'articolo con cui è stato introdotto il concetto, firmato insieme a un biologo che per primo lo aveva coniato, è P. Crutzen, E. F. Stoermer, The Anthropocene, in "IGBP Global Change Newsletter", 41, 2000. In italiano, si veda P. Crutzen, Benvenuti nell'Antropocene! L'uomo ha cambiato il clima, la Terra entra in una nuova era, Mondadori, Milano 2005.

[11] Oltre al sito web ufficiale del progetto (https://theanthropocene.org), si veda il catalogo della mostra (anche in edizione italiana): E. Burtynsky, J. Baichwal, N. de Pencier, Antropocene, 2018.

[12] Cfr. J. R. McNeill, P. Engelke, La Grande accelerazione. Una storia ambientale dell'Antropocene dopo il 1945, Einaudi, Torino 2018 (ed. or. 2013). Sebbene non menzioni il concetto di Antropocene, non ancora introdotto nel dibattito scientifico, ha sottolineato il carattere periodizzante del secondo dopoguerra anche P. Bevilacqua, Il secolo planetario. Tempi e scansioni per una storia dell'ambiente, in C. Pavone (a cura di), '900. I tempi della storia, Donzelli, Roma 1997, pp. 115-149.

[13] Tra i più pessimisti, J. Franzen, E se smettessimo di fingere? Ammettiamo che non possiamo più fermare la catastrofe climatica, Einaudi, Torino 2020 (ed. or. 2019).

[14] S. Neri Serneri, Incorporare la natura. Storie ambientali del Novecento, Carocci, Roma 2005, p. 44.

[15] Per un'esplorazione di lungo periodo sulle malattie epidemiche nel corso della storia, si veda F. M. Snowden, Storia delle epidemie. Dalla morte nera al Covid-19, Leg, Gorizia 2020 (ed. or. 2019). 
[16] Ne ricostruisce un'ampia casistica il bestseller di D. Quammen, Spillover. L'evoluzione delle pandemie, Adelphi, Milano 2014 (ed. or. 2012).

[17] Quammen, 2014, p. 44.

[18] G. Gribaudi, La memoria, i traumi, la storia. La guerra e le catastrofi del Novecento, Viella, Roma 2020, p. 222. In Italia, la ricerca sui disastri "naturali" in età contemporanea è stata lasciata in appannaggio prevalentemente alla sismologia storica: si veda in particolare E. Guidoboni e G. Valensise (a cura di), L'Italia dei disastri. Dati e riflessioni sull'impatto degli eventi naturali 1861 2013, Bononia University Press, Bologna 2013. Va segnalata però una recente riscoperta del tema da parte della storiografia, testimoniata dall'uscita di alcuni lavori importanti, tra i quali si menziona S. Botta, Macerie d'Italia. Storia politica di una nazione in lotta contro la natura, Le Monnier, Firenze 2020, che prende in esame i principali episodi degli ultimi settant'anni.

[19] Cfr. A. Ghosh, La grande cecità. Il cambiamento climatico e l'impensabile, Neri Pozza, Vicenza 2017 (ed. or. 2016).

[20] Sottolinea questa analogia Gribaudi, 2020.

[21] M. Buttino, Samarcanda. Storie di una città dal 1945 a oggi, Viella, Roma 2015, p. 24.

[22] Gli esempi sono tratti da Gribaudi, 2020, pp. 245-248.

[23] S. Botta, Natura imponderabile e umana imprudenza. I terremoti in Italia tra distruzione e ricostruzione, in «Memoria e Ricerca», 2, 2020, p. 367. Per un'analisi storica complessiva sulla gestione delle emergenze nell'Italia repubblicana, si veda Botta, 2020.

[24] Oggetto di una pubblicistica varia ed estesa, il sisma irpino del 1980 è ora al centro di una rinnovata attenzione storiografica: oltre al capitolo a esso dedicato in Botta, 2020, si vedano in particolare i recenti volumi di T. Ricciardi, G. Picone, L. Fiorentino, Il terremoto dell'Irpinia. Cronaca, storia e memoria dell'evento più catastrofico dell'Italia repubblicana, Donzelli, Roma 2020, e di G. I. Moscaritolo, Memorie dal cratere. Storia sociale del terremoto in Irpinia, editpress, Firenze 2020.

[25] P. Bevilacqua, Tra natura e storia. Ambiente, economie, risorse in Italia, Donzelli, Roma 1996, pp. 90-91.

[26] Cfr. per esempio, sul caso italiano, A. Ceroni e I. Ponzi, L'impatto psicologico dei disastri su individui e comunità, in Guidoboni e Valensise (a cura di), 2013, pp. 375-387.

[27] L'intera vicenda è raccontata in T. Merlin, Sulla pelle viva. Come si costruisce una catastrofe. Il caso del Vajont, La Pietra, Milano 1983.

[28] Lo spettacolo è quello di Marco Paolini e Gabriele Vacis, Il racconto del Vajont, presentato come monologo teatrale a partire dal 1993 e trasmesso in diretta televisiva su RaiDue il 9 ottobre 1997.

[29] S. Sontag, Davanti al dolore degli altri, Mondadori, Milano 2003 (ed. or. 2003), p. 15.

[30] Cfr. L. Boltanski, Lo spettacolo del dolore. Morale umanitaria, media e politica, Raffaello Cortina, Milano 2000 (ed. or. 1993). 
[31] Cfr. Gribaudi, 2020, pp. 225-230.

[32] Cr. Gribaudi, 2020, p. 232. Per esempio, il parallelismo tra guerra e terremoto viene facilmente evocato nelle testimonianze dei sopravvissuti al sisma irpino: si veda il film-documentario di Fabrizio Bancale, Terremoto80, 2010. Una raccolta di testimonianze è disponibile online nel sito dell'Archivio multimediale delle memorie (http://www.memoriedalterritorio.it).

[33] Cfr. C. Maier, Un eccesso di memoria?, in «Parolechiave», 9, 1995.

[34] Cfr. Botta, 2020, pp. 239-241.

[35] Cfr. Ligi, 2009, p. 48. 